\title{
Simple EPR/Alanine Dosimeter for Medical Application
}

\author{
Mohamed A. Morsy \\ Chemistry Department, King Fahd University of Petroleum \& Minerals, Dhahran, KSA \\ Email: mamorsy@kfupm.edu.sa
}

Received October 4, 2012; revised November 3, 2012; accepted November 16, 2012

\begin{abstract}
Linac x-ray and direct gamma irradiation sources were used in this study to irradiate simple polycrystalline alaninein-glass ( $\mathrm{AiG}$ ) dosimeters with low-doses, typical for medical therapy, and high-doses, typical for syringes' sterilization processe, respectively. The generated "stable" alanine radicals were quantitatively investigated by electron paramagnetic resonance (EPR) spectroscopy in the presence of an external standard reference $\left(\mathrm{Mn}^{2+} / \mathrm{MgO}\right)$ to correct for spectrometer sensitivity variation. The results indicated that the de-amination of L-alanine is the main reaction to form the "stable" radical and the AiG-dosimeter gives comparable sensitivity for both low and high radiation doses. Moreover, a linear EPR-radiation dose response is observed over a very wide range, from 0 to $50 \mathrm{kGy}$, which is contrary to what has been reported by Bruker BioSpin using alanine's pellet. This linear response and the well-known doses' cumulative characteristics of alanine are in favor of the use of this simple dosimeter in ample medical applications, particularly the conventional radiotherapy treatment per patient.
\end{abstract}

Keywords: Alanine Dosimetry; Radiotherapy Dosimetry; Megavoltage Radiotherapy; Irradiation Doses

\section{Introduction}

Ionizing radiation is commonly used for the sterilization of medical products, pathogen reduction in meats and the disinfestations of imported fruits and vegetables. It is also used in radiation therapy (radiotherapy or x-ray therapy), which uses different forms of radiation like superficial photon beam, megavoltage photon beam, electron beam, or gamma-ray beam. The $\mathrm{x}$-ray beam and electron beam are primarily from linear accelerator (Linac) while gamma-ray beam is from cobalt-60 machine. The exposure to these sources causes biological damage at the atomic level leading to cell death. However, careful utilization and treatment planning can precisely direct the radiation to the cancer while minimizing radiation damage to normal surrounding tissues. This promotes a "radiation dosimetry" field of research to accurately measure the absorbed dose in materials due to the interaction with ionizing radiation [1].

In general, ionizing radiation produces free radicals in many forms of matter that is quantitatively detected by an electron paramagnetic resonance (EPR) spectrometer [2-4]. For example, the amino acid L-alanine forms a very stable free radical when subjected to ionizing radiation that is dose dependent. This dependency has very high accuracy and low error, and has thus become a favored dosimetry technique in Gamma, Electron beam and x-ray irradiation facilities.

The positive characteristics of EPR/alanine dosimetry, such as, stability, reproducibility, cumulatively and independency to the variations in dose rate make it a reliable technique for dose measurements in industrial as well as medical irradiation processes [5,6]. Gel, film, and pellet are three well-known forms of alanine dosimeters, commercially available for the measurements of radiation doses. Many types of binders were used to prepare these forms, e.g., paraffin, acetate polyvinyl, etc. However, the binding material may introduce a background signal or even react with the generated "stable" radicals [7-10]. These activities of the binding materials will definitely influence the EPR measurements of the irradiated alanine at different dose ranges. Alternatively, the use of pure polycrystalline alanine without a binder was proven to be a reliable dosimetric material, however, a limited number of studies [11-14] have been published on its usage so far.

In this study, special attention has been directed to investigate the potential of alnine-in-glass $(\mathrm{AiG})$ dosimeter in different medical applications including radiotherapy. To the best of our knowledge, this work is the first to employ a single type of dosimeter for a wide range of doses from the therapy range or below to the industrial medical related application, namely syringe sterilization. The obtained results indicate that the AiG dosimeter 
show comparable sensitivity for both ranges and an amazed linear EPR-radiation doses' response, which is contrary to what has been reported by Bruker BioSpin using alanine's pellet [15].

\section{Materials and Methods}

\subsection{Alanine Dosimeter}

Alanine-in-glass (AiG) dosimeters were prepared by filling Pyrex glass tubes (length: $40 \mathrm{~mm}$; inner diameter (ID) $>3 \mathrm{~mm}$; wall thickness $>0.5 \mathrm{~mm}$ ) with high purity ( $>99 \%$ ) polycrystalline L- $\alpha$-alanine obtained from Fluka AG, Switzerland and used as received. Cotton was used to keep alanine in place. In all tubes, the height of alanine column was enough to cover the whole active region of the microwave cavity in the EPR spectrometer. The detailed preparation method of $\mathrm{AiG}$ dosimeter and the factors affecting he EPR sampling have been reported previously [11].

\subsection{Irradiation of Dosimeters}

Two different irradiation sources used in medical applications were utilized to irradiate the AiG dosimeters at room temperature. For the radiotherapy, a group of dosimeters was irradiated cumulatively by a $6 \mathrm{MV}$ x-ray beam from a linear accelerator (Clinac $2100 \mathrm{C} / \mathrm{D}$, Varian, USA) with an output of $1 \mathrm{cGy}$ per monitor unit (MU) for doses ranging from 0 to $100 \mathrm{~Gy}$. The dose calibration of this source is performed by ionization chamber. These dosimeters were placed in the geometric center of the field using solid water phantom (Model 547, Gammex-RMI, USA) at its depth of maximum dose $(\sim 1.5 \mathrm{~cm})$. The other group of AiG dosimeters were irradiated by a ${ }^{60}$ Co source (Model IR 103, MDS Nordion, Canada) used by El-Shefaa local industrial plant to sterilize the newly manufactured medical syringe. The source activity was 9.03 PBq (244 kCi) and the dose rate through the irradiation was $0.38 \mathrm{~Gy} / \mathrm{s}$. This group of dosimeter was irradiated along with a calibration standard set that is used from time to time to prove that the appropriate doses have been delivered for sterilization.

\subsection{EPR Measurements}

The EPR spectra of irradiated alanine dosimeters were recorded at room temperature using X-band EPR spectrometer (JEOL, model JES-RE1X) equipped with a standard cylindrical microwave resonator cavity operating in a TE011 mode with a $100 \mathrm{kHz}$ field modulation frequency. The used EPR acquisition parameters were as follows: $10 \mathrm{~mW}$ for microwave power, $1.25 \mathrm{mT}$ for amplitude modulation, a time constant of $100 \mathrm{~ms}$, with 4096 channels for $20 \mathrm{mT}$ width (center field $366 \mathrm{mT}$ ). These parameters compromise between signal-to-noise ratio and resolution. Spectra were collected and analyzed using EWW in EPR software [16].

The dose responses were assessed using the peak-topeak amplitude of the most intense EPR peak, i.e. the middle one in the first-derivative EPR spectrum that was normalized to the average amplitude of the $2^{\text {nd }}$ and $5^{\text {th }}$ $\mathrm{Mn}^{2+}$ lines of the standard reference $\left(\mathrm{Mn}^{2+} / \mathrm{MgO}\right)$. This reference was used to correct sensitivity variations in the spectrometer because of its hyperfine splitting nature (sextet peaks of $a_{\mathrm{Mn}(\mathrm{II})} \sim 6.9 \mathrm{mT}$ ), which, as shown in Figure 1, will not interfere with the middle peaks of alanine radicals (pentet peaks of $a_{\mathrm{Rad}} \sim 2.5 \mathrm{mT}$ ).

\section{Results}

Many samples of the AiG dosimeters were prepared in 3 mm (i.d.) for different irradiation model experiments including radiotherapy and radio-industrial applications. The first group of the dosimeter consists of three samples in sealed, degassed-sealed and unsealed glass tubes that have been irradiated to $25 \mathrm{kGy}$ as shown in Figure 2.

These samples were further investigated for the deamination reaction process of alanine by measuring their Raman spectra against the un-irradiated form (Figure 3).

A second group of the AiG-dosimeter consists of ten samples in unsealed glass tubes were utilized in the dose calibration procedure experiment conducted at El-Shefaa

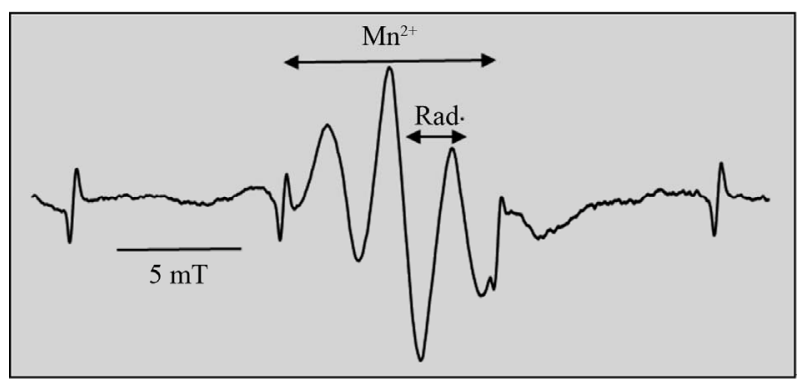

Figure 1. An EPR spectrum of irradiated polycrystalline AiG dosimeter superimpose the $3^{\text {rd }}$ and $4^{\text {th }}$ lines of $\mathrm{Mn}^{2+}$ external reference spectrum.

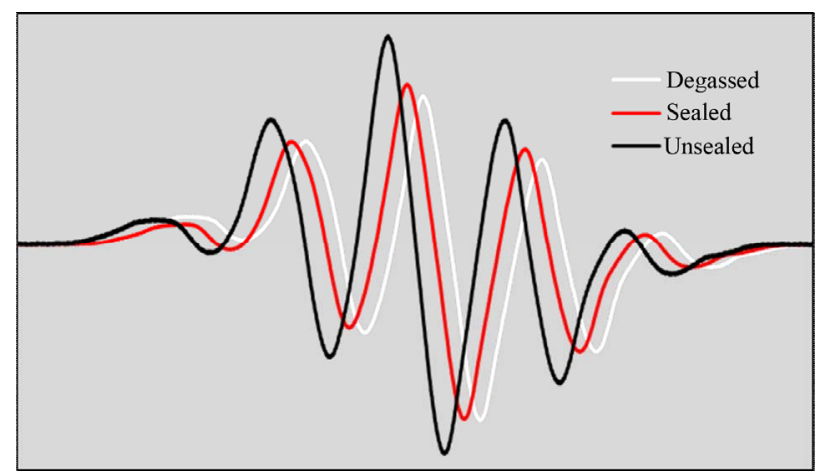

Figure 2. The EPR-signals as a function of the AiG dosimeter forms irradiated to $25 \mathrm{kGy}$. 
local industrial plant for the ${ }^{60} \mathrm{Co}$ irradiation source (Model IR 103, MDS Nordion, Canada). This source sterilizes newly manufactured medical syringe. The presented spectra in Figure $\mathbf{4}$ are samples of those exposed to distinct doses in the range $5-45 \mathrm{kGy}$.

Similar results were obtained for another group of the same dosimeter, which were irradiated cumulatively by a $6 \mathrm{MV}$ x-ray beam from a linear accelerator (Clinac 2100 C/D, Varian, USA) with an output of $1 \mathrm{cGy}$ per monitor unit (MU) for doses ranging from 0 to $100 \mathrm{~Gy}$.

\section{Discussion}

The over modulated EPR spectra in Figure 2 are the characteristic EPR features of alanine, but with different peak-to-peak intensity. The quantitative analysis of these results indicated that the unsealed dosimeter is $25 \%$ to $30 \%$ more intense than the sealed and degassed-sealed dosimeters, respectively. The lowering in the signal intensity of the sealed samples could be attributed to a possible recombination of a fraction from the generated free radicals that did not escape from the sealed tubes and hence reconstruct about quarter or more of the alanine molecular system. For the degassed-sealed dosimeter, the

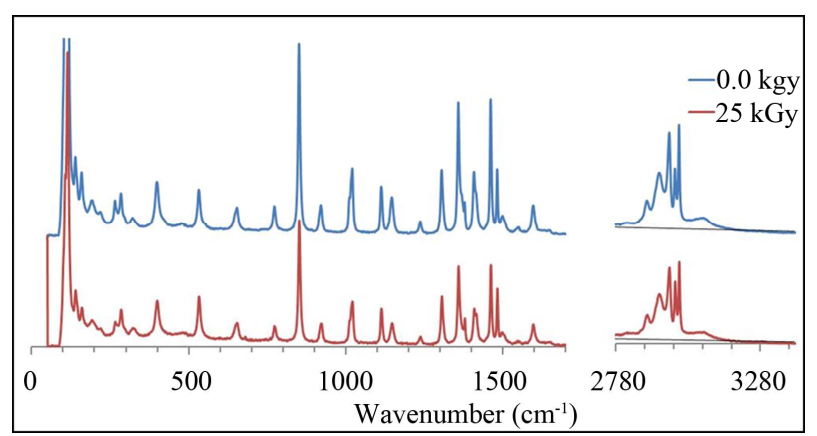

Figure 3. Raman spectra of $\mathbf{2 5}$ kGy irradiated polycrystalline alanine (bottom) and none-irradiated polycrystalline alanine (top).

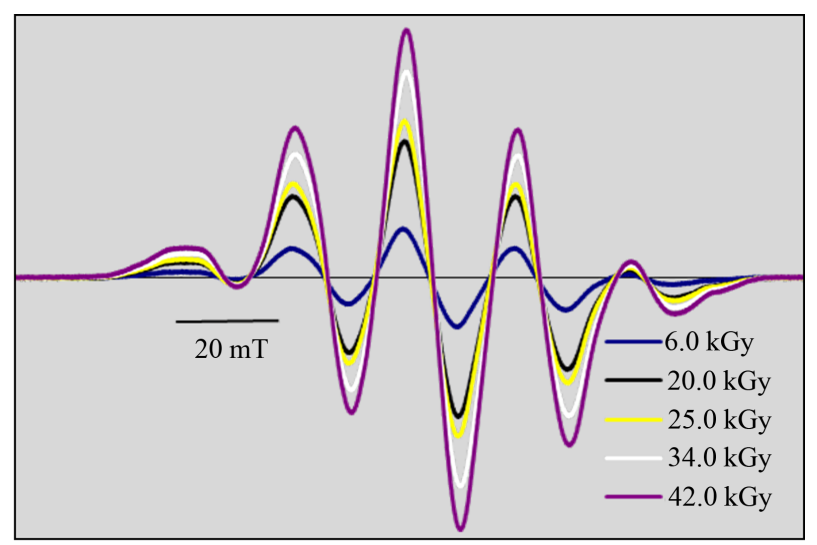

Figure 4. Sample of the EPR spectra of polycrystalline alanine dosimeters irradiated to different doses conducted at the different positions in syringes' sterilization processes. extra lowering is correlated with the absence of competetive reactions between the flying radicals and any of the trapped atmospheric gaseous molecules. These results are in good agreement with the Zagorski and Sehested investigation [17] who observed that the deamination of alanine to form the radical anion $\mathrm{CH}_{3} \mathrm{CHCO}_{2}^{-}$by ionization energy will only affect $80 \%$ of the molecules in time of milliseconds while the remaining $20 \%$ are probably involved more or less in advanced reconstruction of alanine molecule.

Further investigation on the deamination of alanine was conducted by evaluating the Raman spectra in Figure 3. Since Raman spectra provide valuable "fingerprints" for comparing and differentiating materials, the examination of the spectra in Figure 3 provides an ideal technique for assessing the deamination or any trace evidence. The results did not show trace evidence except a decrease in the intensities of certain vibrational modes, such as the bands at $851,1019,1359$ and $1461 \mathrm{~cm}^{-1}$. The comparison between these results and a recent study on the normal vibrational mode assignment of the $\mathrm{L}$-alanine Zwitterion (ALAZW) using density functional theory (DFT) calculations by Kapita et al. [18] as presented in Figure 5.

These results indicated that the observed reduction of the above mentioned modes are directly related to the $\mathrm{C}-\mathrm{N}$ bond or of modes are affected by the deamination process. For example, the band at $851 \mathrm{~cm}^{-1}$, which shows the most remarkable reduction in its intensity in the irradiated alanine sample compare to the un-irradiated one, is attributed to the stretching vibrational mode of N-C

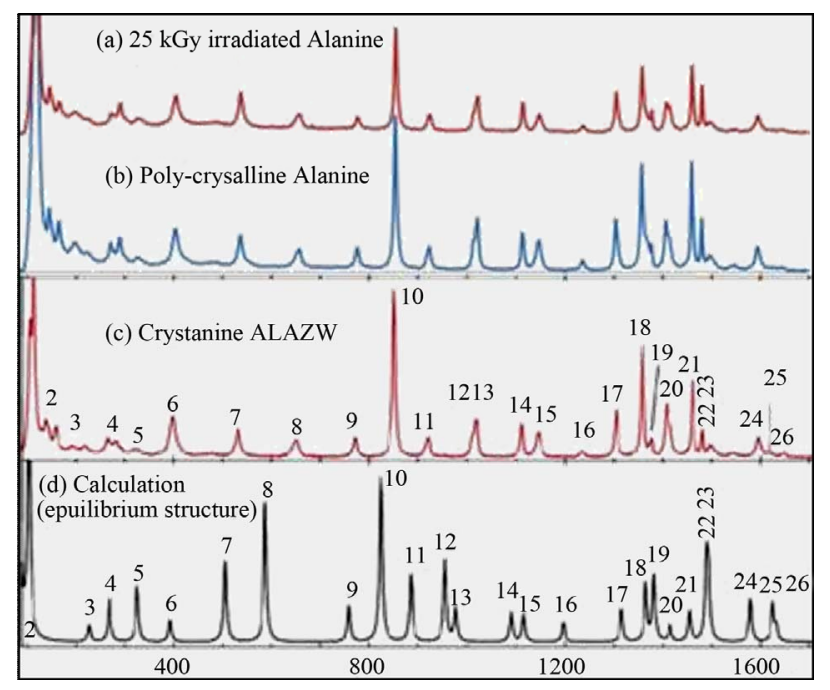

Figure 5. Raman spectra of 25 kGy irradiated polycrystalline alanine (a), none-irradiated polycrystalline alanine (b), crystalline alanine zewitter (ALAZW) ion (c) and the simulated spectrum (d) for ALAZW's equilibrium geometry using the $a b$ initio calculations using the computational level B3LYP/6-31++G**/CPCM in Ref. [18]. 
bond. The other bands are either related to deformation of the $\mathrm{CCN}$ mode, the rocking of the $\mathrm{C}_{\alpha} \mathrm{H}$ mode or the deformation of the $\mathrm{NH}_{3}$ modes, respectively.

These results confirm "deamination reaction of $\mathrm{L}$ alanine" as the main reaction of the irradiated alanine andin the absence of the reconstruction of process, the major reaction scheme of the L-alanine should be:

The proposed irreversibility in Scheme 1 supports the linear dependency of the radical formation responses versus the doses of the energy of ionization. In the previously reported work [11], this dosimeter design confirms the linear response at the low radiation range by showing a very good linear trend in the dose range 0.1 20 Gy with regression coefficient of 0.999 . This promotes us to expand the utilization of the AiG dosimeter in other applications that require very high dose of ionization energy, such as, the calibration of sterilization processes (Figure 4) as well as an important medical application, the radiotherapy, with doses comparable to those in Ref. [11], but using different irradiation source. The industrial sterilization results in Figure 4 show systematic EPR-peak intensity variation as indicated by the figure's legends, which are next to the negative part of the EPR-peaks. For the cumulative doses of a $6 \mathrm{MV}$ $\mathrm{X}$-ray beam from Clinac $2100 \mathrm{C} / \mathrm{D}$ source that is used for radiotherapy treatments a similar faction has been observed, too.

The dependency of the radical formation on the energy of the radiation of the different groups is presented in Figure 6. Assuming this dependency produces perfect trend path through the origin, the linear regression analysis, which attempts to explain this dependency, is validated with high correlation coefficient of 0.996 . This result confirms the novelty of the used $\mathrm{AiG}$ dosimeter and the irreversibility of the radical formation mechanism.

For the radiotherapy, Figure 6 also indicated that the dependency of the radical formation validates the linear trend of the EPR-Dose relation and even with excellent regression coefficient that approaches unity and has

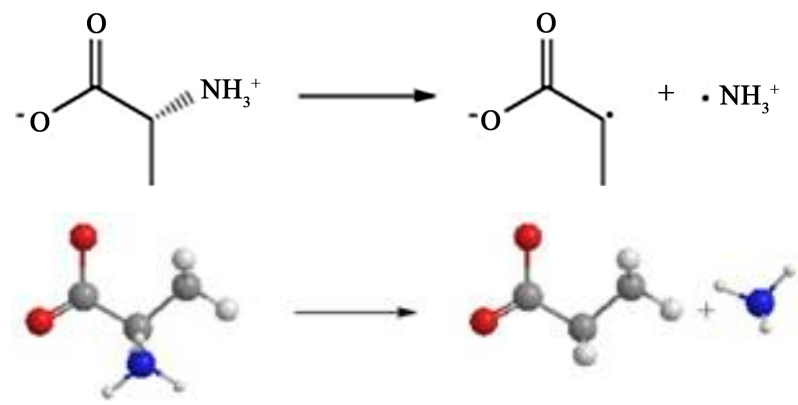

Scheme 1. The de-amination reaction of L-alanine via homolytic bond cleavage process to form "stable" radical anion $\mathrm{CH}_{3} \mathrm{CHCO}_{2}^{-}$by ionization energy.

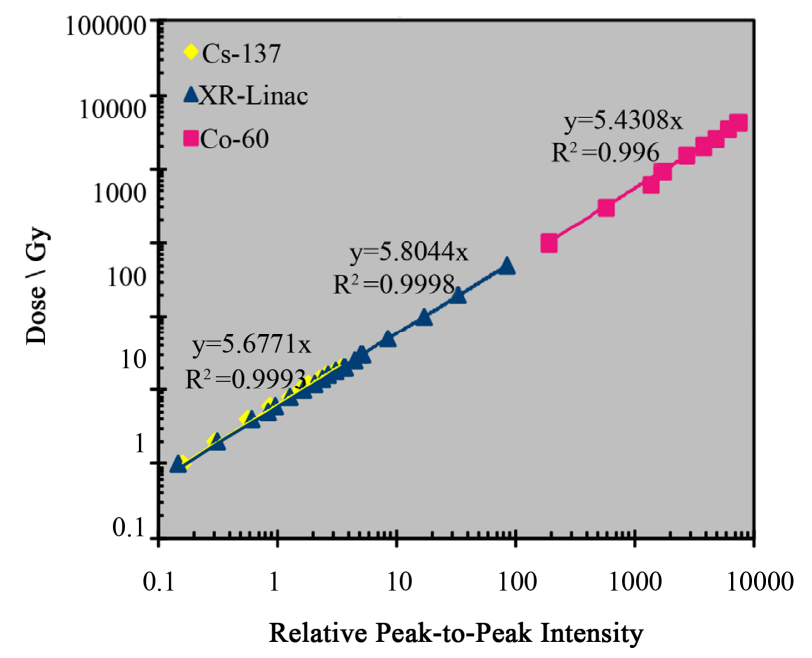

Figure 6. Dose response curve for AiG dosimeters rradiated by different ionizing radiation sources to doses in the range 0.1 Gy - 100 kGy.

comparable slope to the one that has been produced by Cs-137 radiation source (yellow diamond symbols in Figure 6).

Lastly, the EPR-measurements of each dosimeter were recorded several times during a period of one year after irradiation. The results indicated that the dose responsesremained within $1 \%$ of the original measurement for the entire year that proves the high stability of the polycrystalline AiG dosimeters. These results are in good agreement with the early reported characteristics of alanine as a dosimeter, such as the unusual stability of the radiation-induced radicals $[19,20]$, the high radiation sensitiveity [21,22] and the small dependences on ambient conditions, radiation quality and dose rate.

\section{Conclusion}

The used AiG dosimeter show comparable sensitivity for both low and high radiation doses, typical for medical therapy and industrial applications, respectively. It is also confirm the "deamination reaction of L-alanine" is the main process, which, in absence of the reconstruction of alanine molecular system, will produce a prefect EPRradiation dose linear relation. Moreover, this dosimeter was found to be more representative to high radiation doses than the other types of alanine dosimeters, particularly the alanine pellets. The results support the proposal of the usage of $\mathrm{AiG}$ as potential dosimeter in the EPR dosimetry for the clinical gamma irradiation processes (radiotherapy treatment) such as the determination of the radiation dose received by a patient in the brachytherapy who received high doses for intra-oral, pharyngeal or laryngeal malignancies, or total-body irradiation prior to bone marrow transplants (BMT), for example, because of its simplicity and accuracy compare to the TLD method 
or the EPR dentinal enamel studies [23,24]. Moreover, the known cumulative characteristic should support the establishment of a fiducial tracking system for real-time tracking in radiation therapy as well as in many radioindustrial applications such as trends in radiation sterilization of health care products.

\section{Acknowledgements}

The author would like to acknowledge the King Fahd University of Petroleum and Minerals support provided for this work. The author also thanks Mr. Al-Ghamdi (Saudi Aramco Medical Services) and Mr. Farahat (ElShefaa Plant Industry) and Dr. Al-Karmi (Physics Department at KFUPM) for their valuable assistance in the irradiation processes.

\section{REFERENCES}

[1] W. L. McLaughlin, A. W. Boyd, K. Chadwick, J. C. McDonald and A. Miller, "Dosimetry for Radiation Processing," Taylor \& Francis, London, New York, Philadelphia, 1989.

[2] M. Ikeya, J. Miyajima and S. Okajima, "ESR Dosimetry for Atomic Bomb Survivors Using Shell Butons, and Tooth Enamel," Japanese Journal of Applied Physics, Vol. 23, 1984, pp. 679-710. doi:10.1143/JJAP.23.L697

[3] I. Caracelli, M. C. Terrile and S. Mascarenhas, "Electron Spin Resonance Dosimetric Properties of Bone," Health Physics, Vol. 50, No. 2, 1986, pp. 259-263. doi:10.1097/00004032-198602000-00009

[4] K. Sato, "Study of an Asymmetric ESR Signal in X-Irradiated Human Tooth Enamel," Calcified Tissue International, Vol. 29, No. 1, 1979, pp. 95-99. doi:10.1007/BF02408063

[5] M. F. Desrosiers and D. A. Schauer, "Electron Paramagnetic Resonance (EPR) Biodosimetry," Nuclear Instruments and Methods B, Vol. 184, No. 1-2, 2001, pp. 219228. doi:10.1016/S0168-583X(01)00614-0

[6] D. A. Schauer, A. Iwasaki, A. A. Romanyukha, H. M. Swartz and S. Onori, "Electron Paramagnetic Resonance (EPR) in Medical Dosimetry," Radiation Measurements, Vol. 41, No. Supplement 1, 2007, pp. S117-S123. doi:10.1016/j.radmeas.2007.01.006

[7] R. B. Hayes, "Concerns Regarding Recent NIST Publications on Alanine Dosimetry. Comments," Radiation Physics and Chemistry, Vol. 59, No. 4, 2000, pp. 443-444. doi:10.1016/S0969-806X(00)00312-1

[8] M. S. Jahan and K. S. McKinny, "Radiation-Sterilization and Subsequent Oxidation of Medical Grade Polyethylene: An ESR Study," Nuclear Instruments and Methods in Physics Research Section B, Vol. 151, No. 1-4, 1999, pp. 207-212. doi:10.1016/S0168-583X(99)00083-X

[9] M. A. Morsy and M. H. Shwehdi, "Electron Spin Resonance Spectral Study of PVC and XLPE Insulation Materials and Their Life Time Analysis," Spectrochimica Acta Part A, Vol. 63, No. 3, 2006, pp. 624-630. doi:10.1016/j.saa.2005.06.011

[10] W. X. Zhang, H. Wang and J. Z. Sun, "Radiation Effects on the Blends of Polyisobutylene," Journal of Radiation Research and Radiation Processing, Vol. 9, No. 1, 1991, pp. 23-27.

[11] A. M. Al-Karmi and M. A. Morsy, "EPR of Gamma-Irradiated Polycrystalline Alanine-In-Glass Dosimeter," Radiation Measurements, Vol. 43, No. 7, 2008, pp. 13151318. doi:10.1016/j.radmeas.2008.04.088

[12] M. A. Morsy, "An Adaptable EPR/Alanine Dosimeter in Radiotherapy Dose Range," EPRBioDose 2010 International Conference, Mandelieu-La-Napoule, 10-14 October 2010, pp. 102-109.

[13] B. Ciesielski, K. Schultka, A. Kobierska, R. Nowak and Z. Peimel-Stuglik, "In Vivo Alanine/EPR Dosimetry in Daily Clinical Practice: A Feasibility Study," International Journal of Radiation Oncology Biology Physics, Vol. 56, No. 3, 2003, pp. 899-905. doi:10.1016/S0360-3016(03)00196-2

[14] K. Schultka, B. Ciesielski, K. Serkies, T. Sawicki, Z. Tarnawska and J. Jassem, "EPR/Alanine Dosimetry in LDR Brachytherapy: A Feasibility Study," Radiation Protection Dosimetry, Vol. 120, No. 1-4, 2006, pp. 171-175. doi:10.1093/rpd/nci528

[15] Bruker BioSpin, "Alanine Dosimetry," 2012. http://www.bruker-biospin.com/dosimetry0.html

[16] EWWIN 5.21, "For All EPR and ESR Spectrometers, except Bruker ESP300, EMX and Newer Bruker Spectrometers," Scientific Software Services, Northville, 2006.

[17] Z. P. Zagórski and K. Sehested, "Transients and Stable Radical from the Deamination of $\alpha$-Alanine," Journal of Radioanalytical and Nuclear Chemistry, Vol. 232, No. 1-2, 1998, pp. 139-141. doi:10.1007/BF02383729

[18] J. Kapitan, V. Baumruk, V. Kopecky and P. Bour, "Conformational Flexibility of L-Alanine Zwitterion Determines Shapes of Raman and Raman Optical Activity Spectral Bands," The Journal of Physical Chemistry A, Vol. 110, No. 14, 2006, pp. 4689-4696. doi:10.1021/jp060260o

[19] O. F. Sleptchonok, V. Nagy and M. F. Desrosiers, “Advancements in the Accuracy of the Alanine Dosimetry System. Part 1. The Effect of Environmental Humidity," Radiation Physics and Chemistry, Vol. 57, No. 2, 2000, pp. 115-133. doi:10.1016/S0969-806X(99)00338-2

[20] E. Malinen, M. Z. Heydari, E. Sagstuen and E. O. Hole, "Alanine Radicals, Part 3: Properties of the Components Contributing to the EPR Spectrum of X-Irradiated Alanine Dosimeters," Radiation Research, Vol. 159, No. 1, 2003, pp. 23-32. doi:10.1667/0033-7587(2003)159[0023:ARPPOT]2.0.CO;2

[21] V. Nagy, J. M. Puhl and M. F. Desrosiers, "Advancements in Accuracy of the Alanine Dosimetry System. Part 2. The Influence of the Irradiation Temperature," Radiation Physics and Chemistry, Vol. 57, No. 1, 2000, pp. 1-9. doi:10.1016/S0969-806X(99)00339-4

[22] B. Rakvin, "Improvement of Sensitivity in the Alanine/ ESR Dosimetry," Applied Radiation and Isotopes, Vol. 47, No. 5-6, 1996, pp. 965-968 doi:10.1016/0969-8043(96)00004-8

[23] R. Kudynski, J. Kudynska and H. A. Buckmaster, "The 
Application of EPR Dosimetry for Radiotherapy and Radiation Protection," Applied Radiation and Isotopes, Vol. 44, No. 6, 1993, pp. 903-906. doi:10.1016/0969-8043(93)90042-9

[24] B. Pass, R. E. Wood, F. F. Liu, M. McLean and J. E. Al- drich, "High Radiation Doses from Radiotherapy Measured by Electron Spin Resonance in Dental Enamel," $R a$ diation Protection Dosimetry, Vol. 76, No. 4, 1998, pp. 239-247. doi:10.1093/oxfordjournals.rpd.a032269 